TIPA. Travaux interdisciplinaires sur la parole et le langage

$27 \mid 2008$

Apprentissage et acquisition d'une langue seconde

\title{
Liste des publications 2008
}

\section{(2) OpenEdition}

1 Journals

Édition électronique

URL : https://journals.openedition.org/tipa/453

DOI : 10.4000/tipa.453

ISSN : 2264-7082

Éditeur

Laboratoire Parole et Langage

Édition imprimée

Date de publication : 1 juin 2008

ISSN : 1621-0360

Référence électronique

"Liste des publications 2008 », TIPA. Travaux interdisciplinaires sur la parole et le langage [En ligne], 27 |

2008, mis en ligne le 04 décembre 2012, consulté le 08 septembre 2021. URL : http://

journals.openedition.org/tipa/453; DOI : https://doi.org/10.4000/tipa.453

Ce document a été généré automatiquement le 8 septembre 2021.

\section{cc) (†)}

La revue TIPA. Travaux interdisciplinaires sur la parole et le langage est mise à disposition selon les termes de la licence Creative Commons Attribution - Pas d'Utilisation Commerciale - Pas de Modification 4.0 International. 


\section{Liste des publications 2008}

1 ALI, S. Optimising the automatic functional annotation of English intonation. Proceedings of Speech Prosody 2008 (4 : 2008 mai 6-9 : Campinas, BRAZIL). 2008, p. 127-131. [ACT]

BEL, B. Acquisition et représentation de connaissances en musique. Thèse de Doctorat en Sciences (nouveau régime) : Université de Droit, d'Economie et des Sciences d'AixMarseille III. Sous la direction de M. Alain Guénoche. Novembre 1990. Paris: EDILIVRES. 2008, 220 pages Internet : <http://tel.archives-ouvertes.fr/tel-00009692>

BEL, B. Bol Processor - an overview. Proceedings of Symposium Virtual Gamelan Graz: Rules, Grammars, Modeling (2006 octobre 27-28 : Graz, AUSTRIA). Internet <http:// www.lpl.univ-aix.fr/ belbernard/music/BolProcessorOverview/

BolProcessorOverview.pdf $>$ [INV]

BEL, B. Corpus « Ambedkar ». CRDO. 2008. Corpus du Centre de Ressources pour la Description de l'Oral <http://crdo.fr/crdo000011>

BEL, B. Corpus « Grindmill songs ». Corpus du Centre de Ressources pour la Description de l'Oral <http://crdo.fr/crdo000717>

BERTRAND, R. Corpus «CID ». Corpus du Centre de Ressources pour la Description de l'Oral <http://crdo.fr/crdo000027>

7 BERTRAND, R.; BLACHE, P.; ESPESSER, R.; FERRÉ, G.; MEUNIER, C.; PRIEGO-VALVERDE, B.; RAUZY, S. Le CID - Corpus of Interactional Data - Annotation et Exploitation Multimodale de Parole Conversationnelle . Traitement Automatique des Langues, vol. 49, no. 3. 2008 [A paraître], p. 1-30. Internet : <http://hal.archives-ouvertes.fr/ hal-00349893> [ACL]

BERTRAND, R.; PORTES, C. Prosodie dans l'interaction. Forme et fonction des contours montants du français, Laboratoire Lordat (2008 avril 4 : Toulouse, FRANCE). Internet : $<$ http://lpl-aix.fr/ fulltext/3567.pdf> [INV]

BLACHE, P.; PROST, J.-P. A Quantification Model of Grammaticality. Proceedings of CSLP08 - Constraint Solving and Language Processing (Hamburg, GERMANY). Hamburg: Roskilde University Press. 2008, p. 5-19. Internet : <http://lpl-aix.fr/ fulltext/3355.pdf> [ACT] 

corpora. Proceedings of LREC08 - Language Resource and Evaluation COnference (2008 : Marrakech, MOROCCO). Marrakech: ELDA. 2008, p. 1-5. Cederom. Internet <http:// hal.archives-ouvertes.fr/hal-00353722> [ACT] exposure, prime-target relationship, and task demands. Memory \& Cognition, vol. 36 no. 4.2008 , p. 828-898. [ACL]

CHENTIR, A.; GUERTI, M.; HIRST, D. Classification by discriminant analysis of energy in view of the detection of accented syllables in standard Arabic. Journal of Computer Science, vol. 4, no. 8. 2008, p. 668-673. ISSN 1807-4545. [ACL]

CHO, H.; RAUZY, S. Study on the phonetic pitch movement of the accentual phrase in Korean read speech . Proceedings of Speech Prosody (4 : 2008 mai 6-9 : Campaninas, BRAZIL). 2008, p. 123-126. Internet : <http://hal.archives-ouvertes.fr/hal-00285529> [ACT]

CORREARD, $\mathrm{N}$. Troubles émotionnels et perturbation du raisonnement déductif. Thèse de doctorat de Psychologie : Université Aix-Marseille 1. Sous la direction de Claude BASTIEN. Décembre 2008.

COYE DE BRUNELIS, T. Allocation dynamique de fonctions : expérimentation et bases méthodologiques pour la conception de systèmes Homme-Machine. Thèse de doctorat de Psychologie : Aix-Marseille 1. Sous la direction de Claude BASTIEN. Décembre 2008.

D'IMPERIO, M.; MICHELAS, A.; PYNTE, J. Can accentual phrase boundaries remove temporary lexical ambiguity in French? Poster. Conference on Human Sentence Processing (CUNY 2008) (2008 mars 13-15 : Chapel Hill, North Carolina, UNITED STATES OF AMERICA).

RAPOSO DE MEDEIROS, B.; D'IMPERIO, M.; ESPESSER, R. La voyelle nasale en PB et son appendice nasal : étude acoustique et aérodynamique. Actes, Journées d'Etude sur la Parole (JEP) (27 : 2008 juin 9-13 : Avignon, FRANCE). 2008, p. 285-288. Internet : <http:// hal.archives-ouvertes.fr/hal-00380694> [ACT]

D'IMPERIO, M.; MICHELAS, A.; PYNTE, J. Can accentual phrase boundaries influence lexical access in French? Poster. Experimental and Theoretical Advances in Prosody. Conference on Prosody and Language Processing (2008 avril 11-13 : Ithaca, NY, UNITED STATES OF AMERICA).

D'IMPERIO, M.; CANGEMI, F.; BRUNETTI, L. The phonetics and phonology of contrastive topic constructions in Italian. Proceedings of Tone and Intonation in Europe $(3: 2008$ septembre 15-17 : Lisbonne, PORTUGAL). [COM]

GILI FIVELA, B.; D'IMPERIO, M. High peak vs high plateau in the identification of contrastive accents in Italian. Poster. Tone and Intonation in Europe (3: 2008 septembre 15-17 : Lisbonne, PORTUGAL).

DUCROT, S.; LÉTÉ, B. Attention et contrôle oculaire en lecture experte. In Michaël, G.A. (ed.) Les dimensions de l'attention visuelle. -- SOLAL. Marseille: SOLAL. 2008, p. 229-264. [OS]

DUSAUTOIR, M.; CASALIS, S.; DUCROT, S. Morphemic units as processing units in beginning readers' visual word recognition : Evidence from initial fixation position. Proceedings of International Conference on Mental Lexicon (sixth : 2008 octobre 7-10 : Banff, CANADA) [Forthcoming]. [ACT]

TIPA. Travaux interdisciplinaires sur la parole et le langage, 27 | 2008 
DUCROT, S.; GHIO, A.; LÉTÉ, B. Logiciel ADIVA (Aide au DIagnostic des déficits VisuoAttentionnels chez l'enfant). (2008)

CASALIS, S.; COLÉ, P.; DUCROT, S. Morphological priming in visual word recognition in French third and fifth graders : Frequency effects. Proceedings of International conference on the mental lexicon ( $6: 2008$ octobre $7-10:$ Banff, Alberta, CANADA) [Forthcoming]. [ACT]

VAISSIÈRE, J.; DUEZ, D.; KAWAGUCHI, Y.; BOULAKIA, G. De la voix et du sens-autour de l'œuvre d'Ivan Fonagy. Actes, Temps, espaces, langages: la Hongrie à la croisée des disciplines (2006 décembre 7-9 : Paris, FRANCE). Paris: P.Renaud. 2008 [A paraître]. Internet : <http://lpl-aix.fr/ fulltext/3149.pdf> [OS]

DUEZ, D. Les troubles de la parole dans la maladie de Parkinson: quelques faits de dysrythmie. Actualités psychologiques, vol. 21, no. 21. 2008, p. 139-156. [ACL]

DUEZ, D.; LEGOU, T.; VIALLET, F. Final Lengthening in Parkinsonian French Speech. Proceedings of International Seminar on Speech Production (2008 décembre 8-12 : Strasbourg, FRANCE). 2008, p. 189-192. Internet : <http://hal.archives-ouvertes.fr/ hal-00364906> [ACT]

DUFOUR, S. Phonological priming in auditory word recognition: When both controlled and automatic processes are responsible for the effects. Canadian Journal of Experimental Psychology, vol. 62. 2008, p. 33-41. [ACL]

ASTÉSANO, C.; ESPESSER, R.; ROSSI-GENSANE, N. Quelques cas particuliers de détachement à gauche - ou la prosodie à l'aide de la syntaxe. Actes, Journées d'Etude sur la Parole (JEP) (27 : 2008 juin 9-13 : Avignon, FRANCE). 2008, p. 109-112. Internet : $<$ http://hal.archives-ouvertes.fr/hal-00380695> [ACT]

BABAULT, S.; FARACO, M. Mobilité et prise de notes: une approche interculturelle. In DERVIN Fred ; BYRAM Michaël (eds.) ISBN 978-2-296-06149-1. Paris, France: L'Harmattan, Collection Logiques sociales. 2008, p. 93-116. [OS]

31 FARACO, M.; KIDA, T. 13. Gesture and the negiotiation of meaning in a second language classroom. In Steven G. McCafferty, Gale Stam (eds.) Gesture. [Second Language Acquistion and Classroom Research] -- 1ère ISBN 0-8058-6053-3. New York and London (Routledge): Laurence Erlbaum Associates. 2008, vol.1 : Part V : Gesture and the L2 Classroom, p. 280-297. [OS]

2 FOUCART, A. Grammatical gender processing in French as a first and a second language. (Le traitement du genre grammatical en francais langue première et seconde). Thèse de doctorat en Psycholinguistique : Universite Aix-Marseille I / University of Edinburgh. Sous la direction de Dr C. Frenck-Mestre (CNRS- U. Provence), Dr H. Branigan (reader, U. Edinburgh), Dr E. Bard (reader, U. Edinburgh. Février 2008. 258 p.

33 OSTERHOUT, L.; POLIAKOV, A.; INOUE, K.; MCLAUGHLIN, J.; VALENTINE, G.; PITKANEN, I.; FRENCK-MESTRE, C.; HIRSCHENSOHN, J. Second-language learning and changes in the brain. Journal of Neurolinguistics, vol. 21, no. 6. 2008, p. 509-521. Internet : <http:// hal.archives-ouvertes.fr/hal-00387578> [ACL]

FRENCK-MESTRE, C.; OSTERHOUT, L.; MCLAUGHLIN, J.; FOUCART, A. The effect of phonological realization of inflectional morphology on verbal agreement in French: Evidence from ERPs. Acta Psychologica, vol. 128. 2008, p. 528-536. [ACL] 
OSTERHOUT, L.; FRENCK-MESTRE, C. How the brain changes during the earliest stages of second language learning. American Association of Applied Linguistics (2008 mars 29-avril 1 : Washington D.C., UNITED STATES OF AMERICA). [INV]

LAINÉ, F.; TARDIF, C.; GEPNER, B. Amélioration de la reconnaissance et de l'imitation d'expressions faciales chez des enfants autistes grâce à une présentation visuelle et sonore ralentie. Annales Médico-Psychologiques, vol. 166. 2008, p. 533-538. Internet : $<$ http://lpl-aix.fr/ fulltext/3198.pdf> [ACL]

GEPNER, B. From neuronal to human communication disorders: A novel approach to autism. Interactions, vol. 1, no. 1. 2008, p. 1-25. [ACL]

GEPNER, B. Une nouvelle approche de l'autisme: des désordres de la communication neuronale aux désordres de la communication humaine. Interactions, vol. 1, no. 1. 2008, p. 1-26. [ACL]

POUCHOULIN, G.; FREDOUILLE, C.; BONASTRE, J.-F.; GHIO, A.; GIOVANNI, A. Analyse Phonétique dans le Domaine Fréquentiel pour la Classification des Voix Dysphoniques. Actes, Journées d'Etude sur la Parole (JEP) (27 : 2008 juin 9-13 : Avignon, FRANCE). Avignon: LIA. 2008, p. 221-224. Internet : <http://hal.archives-ouvertes.fr/ hal-00292400> [ACT]

GHIO, A.; GIOVANNI, A.; TESTON, B.; RÉVIS, J.; YU, P.; OUAKNINE, M.; ROBERT, D.; LEGOU, T. Bilan et perspectives de quinze ans d'évaluation vocale par méthodes instrumentales et perceptives. Actes, Journées d'Etude sur la Parole (JEP) $(27: 2008$ juin 9-13 : Avignon, FRANCE). Avignon: LIA. 2008, p. 309-312. Internet : <http://hal.archivesouvertes.fr/hal-00292401> [ACT]

41 GHIO, A. Complementary Approaches for voice disorder assessment. Proceedings of Congresso Internacional de Fonetica e Fonologia (4:2008 novembre 24-26 : Niteroi, BRAZIL). Niteroi: SBF. 2008, p. 24. Internet : <http://aune.lpl.univ-aix.fr/ ghio/doc/ Bib-2008-SFBNiteroi-Ghio.pdf> [INV]

POUCHOULIN, G.; FREDOUILLE, C.; BONASTRE, J.-F.; GHIO, A.; GIOVANNI, A. Dysphonic Voices and the $0-3000 \mathrm{~Hz}$ Frequency Band. Proceedings of INTERSPEECH 2008 (9: 2008 septembre 22-26 : Brisbane, AUSTRALIA). Brisbane: ISCA. 2008, p. 2214-2217. Internet : <http://aune.lpl.univ-aix.fr/ ghio/doc/Bib-2008-interspeech-pouchoulin.pdf> [ACT]

3 GIOVANNI, A.; RÉVIS, J.; GHIO, A. Le phoniatre face à l'évaluation instrumentale des dysphonies. Actes, Congrès de la Société Française de Phoniatrie (64 : 2008 octobre 14 : Paris, FRANCE). Paris: SFP. 2008. [INV]

44 KIDA, T. Does gesture aid discourse comprehension in the L2? In Steven G. McCafferty, Gale Stam (eds.) Gesture. [Second Language Acquistion and Classroom Research] ISBN 0-8058-6053-3. New York and London (Routledge): Lawrence Erlbaum Associates. 2008 : Gesture and Communication in the L2, p. 131-156. [OS]

KRIEGEL, S.; LUDWIG, R.; HENRI, F. Encoding path in Mauritian Creole and Bhojpuri. Problems of language contact. Creole Structures between substrates and superstrates (2005 juin 2-5 : Leipzig, GERMANY). In Michaelis, S. / Benjamins (ed.) Roots of Creole Structures. Weighing the contribution of substrates and superstrates. (Creole Language Library; 33) ISBN 9789027252555. Amsterdam/Philadelphia: John Benjamins. 2008 : Encoding path in Mauritian Creole and Bhojpuri. Problems of language contact, p. 169-196. [OS] 

conversationnelle. Actes, Journées d'Etude sur la Parole (JEP) (27 : 2008 juin 9-13 : Avignon, FRANCE). 2008, p. 97-100. Internet : <http://hal.archives-ouvertes.fr/ hal-00292408> [ACT]

Conference on Speech Prosody (Fourth : 2008 mai 6-9 : Campinas, BRAZIL). 2008, p.
567-570. Internet : <http://hal.archives-ouvertes.fr/hal-00285645> [ACT]

francophones. Thèse de doctorat de Sciences du Langage : Université Aix-Marseille I. Sous la direction de Noël Nguyen. Septembre 2008. Internet $:<h t t p: / / t e l . a r c h i v e s-$ ouvertes.fr/tel-00334980/fr/>

59

MORTREUX, S. The production of English coronal consonants by French learners of English. Actes, Didactique des langues et linguistique, Colloque international d'étudiants chercheurs (2006 juillet 4-7 : Grenoble, FRANCE). Grenoble: PUG. 2008, p. 87-93. [ACT]

TIPA. Travaux interdisciplinaires sur la parole et le langage, 27 | 2008 


\section{aires du Laboratoire Parole et Langage (TIPA), no. 26. 2008, p. 93-113. Internet : <http://hal.archives-ouvertes.fr/hal-00292399>} [SLC]

MORTREUX, S. English coronal consonants produced by L2 French learners. An articulatory and acoustic study. Proceedings of International Seminar on Speech Production (2008 décembre 8-12 : Strasbourg, FRANCE). 2008, p. 145-148. [ACT]

NGUYEN, N.; FAGYAL, Z. Acoustic aspects of vowel harmony in French. Journal of Phonetics, vol. 36, no. 1. 2008, p. 1-27. Internet : <http://hal.archives-ouvertes.fr/ hal-00256384> [ACL]

NIEBUHR, O.; LANCIA, L.; MEUNIER, C. On place assimilation in French sibilant sequences. Proceedings of International Seminar on Speech Production (8 : 2008 décembre 8-12 : Strasbourg, FRANCE) [Forthcoming]. [ACT]

NISHINUMA, Y. Book Review : Principles of Phonetic Sciences by Osamu Fujimura. Revue Monthly Gengo (Monthly Journal of Languages), vol. 37, no. 5. 2008, p. 110. ISSN 0287-1696. [AP]

NISHINUMA, Y. Book Review - Principles of Phonetic Sciences by Osamu Fujimura. Revue Monthly Gengo (Monthly Journal of Languages), vol. 37, no. 5. 2008, p. 110. ISSN 0287-1696. Internet : <http://lpl-aix.fr/ fulltext/3321.pdf> [AP]

NISHINUMA, Y. Sounds in Provence. Revue Monthly Gengo (Monthly Journal of Languages), vol. 37, no. 7. 2008, p. 4-5. ISSN 0287-1696. Internet : <http://lpl-aix.fr/ fulltext/3322.pdf> [AP]

NISHINUMA, Y. Dialogue français (role-play). Corpus du Centre de Ressources pour la Description de l'Oral <http://crdo.fr/crdo000738>

YABE, H.; NISHINUMA, Y.; HAYASHI, A.; LIN, H.; LENG, L. How do non-native speakers perceive Japanese phrase final prosody? An experiment on the perception of pitch and rhythm by Chinese and German subjects . Proceedings of International Symposium on Japanese Language Teaching and Japanese Studies (1 : 2008 décembre 12-15 : Guangdong, CHINA). Guangdong, China: Guangdong University of Foregin Studies. 2008, p. 124-125. Internet : <http://hal.archives-ouvertes.fr/hal-00387569> [ACT]

PALLAUD, B. Les amorces de mot dans la parole spontanée sont-elles des ellipses ? In Jean Claude Pitavy (ed.) Ellipse et effacement. Du schème de phrase aux règles discursives: enjeux du constituant inarticulé et de la place vide. Saint-Etienne: Presses universitaires de Saint-Etienne (PUSE). 2008, p. 259-266. Internet : <http://hal.archivesouvertes.fr/hal-00308391> [OS]

PALLAUD, B.; XUEREB, R. Les troncations et les répétitions de mots chez un locuteur PETITJEAN, C. Représentations linguistiques et accents régionaux du français. Journal of Language Contact, vol. Varia 1, no. 1. 2008. ISSN 1955-2629. [ACL]

PETRONE, C. Le rôle de la variabilité phonétique dans la représentation des contours intonatifs et de leur sens. Thèse de doctorat de Sciences du Langage : Université AixMarseille I. Sous la direction de Mariapaola D'Imperio, CNRS/U. de Provence. 122008.

PROST, J.-P. Modélisation de la gradience syntaxique par analyse relâchée à base de contraintes -- Modelling Syntactic Gradience with Loose Constraint-based Parsing. Thèse en Cotutelle de doctorat en Informatique : Université de Provence et Macquarie University, Sydney, Australia. Sous la direction de Philippe Blache et Diego Molla Aliod, et la direction adjointe de Mark Dras. Décembre 2008. 
BLACHE, P.; RAUZY, S. Influence de la qualité de l'étiquetage sur le chunking: une corrélation dépendant de la taille des chunks. Actes, Traitement Automatique des Langues Naturelles (15 : 2008 juin 9-13 : Avignon, FRANCE) [A paraitre]. Cederom. Internet : <http://hal.archives-ouvertes.fr/hal-00285642> [ACT]

NESTERENKO, I.; RAUZY, S. Utilisation des grammaires probabilistes dans les tâches de segmentation et d'annotation prosodique. Actes, Journées d'Etudes sur la Parole (27 : 2008 juin 9-13 : Avignon, FRANCE) [A paraître]. Cederom. Internet : <http:// hal.archives-ouvertes.fr/hal-00285643> [ACT]

ADER, M.; BLACHE, P.; RAUZY, S. Prise en charge des troubles de la communication : La Plateforme de Communication Alternative . Interactions, vol. 1. 2008, p. 1-19. Cederom. Internet : <http://hal.archives-ouvertes.fr/hal-00285644> (mars 2008) [ACL]

6 ROMAIN, C. Description de la violence verbale en situation difficile d'enseignement. Colloque international De l'impolitesse à la violence verbale (2005 mai 11-13 : Avignon, FRANCE). In N. Auger, B. Fracchiolla, C. Moïse, C. Schultz-Romain (eds.) Des perspectives historiques aux expériences éducatives. Paris: Espaces discursifs - Thierry Bulot - L'Harmattan. 2008, vol.2, p. 97-120. [OS]

ROMAIN, C.; LEBRUN, M. Echange culturel et évolution du rapport à la culture chez des élèves de 6 ans. Actes, Problématiques culturelles dans l'enseignement-apprentissage des langues-cultures, mondialisation et individualisation : approche interdisciplinaire (2008 mai 8-10 : Tallinn, ESTONIA). Tallinn - Estonie: Synergie Pays riverains de la Baltique. 2008 [A paraître]. [ACT]

ROMAIN, C. Sociocultural environments and control of narrative tools at French pupils from 9 to 14 years. Writing Research Across Borders (2008 février 22-24 : Santa Barbara, UNITED STATES OF AMERICA). Santa Barbara - Etats-Unis: Université de Californie Santa Barbara, Etats-Unis. 2008. [COM]

AUGER, N.; FRACCHIOLLA, B.; MOISE, C.; ROMAIN, C.(ed.) La violence verbale. Espaces politiques et médiatiques. (Collection Espaces discursifs). Paris: L'Harmattan. 2008, vol. 1, 231 pages. [DO]

AUGER, N.; FRACCHIOLLA, B.; MOISE, C.; ROMAIN, C.(ed.) Des perspectives historiques aux expériences éducatives. (Collection Espaces discursifs). Paris: L'Harmattan. 2008, vol.2, 288 pages. [DO]

81 ROMAIN, C. Compétences linguistiques et correspondance scolaire internationale. Séminaire Didactique de la littérature en milieu résistant (dir. M. Lebrun). (2008)

B2 ROSSIGNOL, C. Special Needs Institutions. Description, Recording and Analysis of Interventions and Services: Formal Model and Practical Applications . Interactions, vol. 1, no. 1. 2008, p. 1-16. [ACL]

33 SITBON, L.; BELLOT, P.; BLACHE, P. Eléments pour adapter les systèmes de recherche d'information aux dyslexiques. Traitement Automatique des Langues, vol. 48, no. 2. 2008, p. 123-147. Internet : <http://hal.archives-ouvertes.fr/hal-00311305> [ACL]

84 SITBON, L.; BELLOT, P.; BLACHE, P. Lisibilité et recherche d'information : vers une meilleure accessibilité. Intégration de la lisibilité au calcul de la pertinence. Actes, CORIA08 - Conférence en Recherche d'Information et Applications (Trégastel, FRANCE). 2008. Cederom. Internet : <http://lpl-aix.fr/ fulltext/3359.pdf> [ACT]

85 SITBON, L.; BELLOT, P.; BLACHE, P. A corpus of real-life questions for evaluating robustness of QA systems. Proceedings of LREC08 - Language Resource and Evaluation 
Conference (Marrakech, MOROCCO). Marrakech: ELDA. 2008. Cederom. Internet : $<$ http://lpl-aix.fr/ fulltext/3360.pdf> [ACT]

SITBON, L.; BELLOT, P.; BLACHE, P. Evaluation of lexical resources and semantic networks on a corpus of mental associations. Proceedings of LREC08 - Language Resource and Evaluation Conference (Marrakech, MOROCCO). Marrakech: ELDA. 2008. Cederom. Internet : <http://lpl-aix.fr/ fulltext/3361.pdf> [ACT]

STEUCKARDT, A. Les dictionnaires informatisés : un atout pour l'histoire du lexique. Actes, Congrès mondial de linguistique française (2008 juillet 9-12 : Paris, FRANCE). Paris: EDP Sciences. 2008. Cederom. Internet $:<h t t p: / / d x . d o i . o r g / 10.1051 /$ cmlf08151> [ACT]

TELLIER, M. The effect of gestures on second language memorisation by young children. Gesture, vol. 8, no. 2. 2008, p. 219-235. Internet : <http://hal.archivesouvertes.fr/hal-00375251> [ACL]

TELLIER, M. Dire avec des gestes. Le français dans le monde: Recherche et Apllications, no. 44. 2008, p. 40-50. ISSN 0015-9395. Internet : <http://hal.archives-ouvertes.fr/ hal-00375252> [ACL]

TESTON, B. A la poursuite du signal de parole: suite et fin. Actes, Journées d'Etude sur la Parole (JEP) (2008 juin 9-13 : Avignon, FRANCE). Avignon: Université d'Avignon. 2008, p. 397-400. Internet : <http://hal.archives-ouvertes.fr/hal-00308393> [ACT]

91 TESTON, B. Dossier de consultation pour l'aménagement des plates formes techniques du Laboratoire Parole et Langage sur le site « Pasteur » de l'Université de Provence. Aix en Provence 2008, p. 1-76 Internet : <http://lpl-aix.fr/ fulltext/3317.pdf>

TESTON, B. La nasalité dans la phonétique expérimentale : aspects historiques et méthodologiques. Diaporama. Dynamique de la nasalité (2008 septembre 12-16 : Porquerolles, FRANCE). 2008. Cederom.

TORTEL, A. ANGLISH. Corpus du Centre de Ressources pour la Description de l'Oral <http://crdo.fr/crdo000731> (En cours de publication)

TRIM, R. Les réseaux conceptuels au sein de l'évolution historique de la métaphore dans la littérature médiavale anglaise: une approche cognitive. Bulletin de la Société de Stylistique Anglaise, vol. 31. 2008, p. 117-134. [ACL] 